\title{
Application of a central composite face-centered design in the optimization of an Archimedean hydrokinetic turbine
}

\author{
J. Betancour ${ }^{1}$, L. Velásquez ${ }^{1}$, L.Y. Jaramillo ${ }^{2}$, E. Chica ${ }^{1}$, A. Rubio-Clemente ${ }^{1,2}$ \\ ${ }^{1}$ Grupo de Investigación Energía Alternativa, Facultad de Ingeniería, Universidad de Antioquia UdeA, Calle 70, No. 52-21, \\ Medellín, Colombia. \\ ${ }^{2}$ Facultad de Ingeniería, Tecnológico de Antioquia-Institución Universitaria TdeA, Calle 78b, No. 72A-220, Medellín, \\ Colombia. \\ Phone/Fax number: +0057 2195547, e-mail: ainhoarubioclem@gmail.com
}

\begin{abstract}
Currently, in the literature, there are no general guidelines for the optimal hydraulic design of Archimedean screw turbines (AST) used in hydrokinetic applications. Therefore, this study is aiming at selecting the most significant geometric factors, such as the diameter ratio between the inner $\left(D_{i}\right)$ and the outer $\left(D_{o}\right)$ diameters (i.e., $\left.D_{i} / D_{o}\right)$, the axle length $(L)$ and the blade stride $(p)$, influencing the AST performance by using a central composite face-centered (CCF) experimental design combined with the response surface methodology (RSM). The statistical analysis of variance (ANOVA) test identified with a significance level of 0.05 that the most significant variables on the performance of the turbine were $p$ and $D_{i} / D_{o}$. The AST efficiency was evaluated by means of the power coefficient $\left(C_{P}\right)$, which was calculated by means of computational fluid dynamics (CFD) methods coupled with the 6-degrees of freedom (6-DoF) approach. The second-order polynomial model was used to predict the $C_{P}$ and the coefficient of determination $\left(R^{2}\right)$ was found to be $97.4 \%$.
\end{abstract}

Key words. 6-DoF, response surface methodology, Archimedean screw hydrokinetic turbine, central composite design, numerical optimization.

\section{Introduction}

An Archimedean screw turbine (AST) can be implemented for a hydrokinetic application due to its lower installation, maintenance and operating costs, high efficiency and environmental advantages in comparison with alternative hydrokinetic systems used to convert the energy from naturally flowing water (streams, rivers, tidal estuaries, ocean currents) into electricity, which in turn can be used in rural areas and developing countries $[1,2,3]$.

Generally, hydrokinetic turbines operate at much lower energy extraction efficiency than large hydraulic turbines. In this regard, it is important to optimize the rotor design of hydrokinetic turbines to maximize their efficiency. An AST consists of a helical surface (flights) surrounding a central cylindrical shaft. The geometry of the AST can be defined based on several geometric parameters, including the outer and the inner diameters $\left(D_{o}\right.$ and $D_{i}$, respectively), the number of flights $(\mathrm{N})$, the blade stride $(p)$, the blade inclination with respect to the longitudinal axis of the screw $(\alpha)$ and the axle length $(L)$. The cylindrical shaft can be inclined or aligned with the flow. If the cylindrical shaft is tilted, the angle of inclination is called $\beta[3,4]$.

The design of an AST involves, simultaneously, the optimization of geometric parameters, as well as operating variables, so that the power coefficient $\left(C_{P}\right)$ is maximized. However, due to AST is a new and growing technology, there are few guidelines regarding the optimization of the turbine design and performance [5].

In the literature, the discussion concerning the effect of geometric parameters on the performance of an AST for hydrokinetic applications is sparsely explored [4]. Only few references regarding the hydrodynamic effects on the selection of the values for hydrokinetic applications are reported for these parameters $[6,7,8]$.

Traditionally, optimization purposes have been carried out considering the influence of one factor at a time. Nevertheless, this approach does not consider the possibility that the used variables can interact with the others. Thus, in this study, the effect of the three design parameters on the AST performance using the design of experiments (DoE) tool is discussed. In this regard, the relationships between the $C_{P}$ and the design parameters, such as the diameter ratio $\left(D_{i} / D_{o}\right), L$ and $p$, were obtained utilizing a central composite face-centered (CCF) experimental design combined with a secondorder response surface methodology (RSM) and computational fluid dynamics (CFD) analysis.

\section{Materials and methods}

CCF experimental design and RSM allow to study the relationships between the considered geometric design variables $\left(D_{i} / D_{o}, L\right.$ and $\left.p\right)$ on the $C_{P}$ response variable. Statistical analysis of variance (ANOVA) was carried out to identify the adequacy of the developed regression model and to determine the degree of importance of the selected variables. It is highlighted that RSM is able to 
recognize the interaction effects and the influence of the geometric parameters on the $C_{P}$, which was calculated as expressed in Eq. (1) [2].

$$
C_{p}=\frac{P}{0.5 \rho A V^{3}}
$$

where $P$ is the power produced by the turbine, which is equal to the product between the torque generated $(T)$ by the fluid on the turbine and the turbine rotation velocity $(\omega)$. In turn, $\rho$ is the fluid density, $V$ is the flow velocity and $A$ is the swept area that is perpendicular to the flow. In turn, $C_{P}$ is conventionally represented as a function of the tip speed ratio (TSR), which can be obtained by using Eq. (2) $[2]$.

$$
T S R=\frac{\omega R}{V}
$$

where $R$ is the outer radius of the turbine, TSR is the ratio between the tangential speed of the blade at its tip divided by the speed of water.

In the current study, $C_{P}$ was represented by a second-order polynomial model, whose general form is shown in Eq. (3) [9].

$$
\begin{aligned}
Y=\beta_{0}+\sum_{i=1}^{n} \beta_{i} X_{i} & +\sum_{j=1}^{n} \beta_{i i} X_{i}^{2} \\
& +\sum_{i=1}^{n-1} \sum_{i=2}^{n} \beta_{i j} X_{i} X_{j}+\varepsilon
\end{aligned}
$$

where $Y$ is the predicted response; $\beta_{0}$ is the regression coefficient intercept; $\beta_{i}, \beta_{i i}$ and $\beta_{i j}$ represent the linear, quadratic and interaction terms, respectively; $X_{i}$ and $X_{j}$ are the coded independent variables for the design parameters; $n$ is the number of variables; and $\varepsilon$ is the statistical error [9].

The independent variables $\left(D_{i} / D_{o}, L\right.$ and $\left.p\right)$ were varied over 3 levels; i.e., a low, medium and a high level. In Table I, the values of the factors and their corresponding coded levels are shown. It is noteworthy to mention that CCF experimental design is a well-renowned method in RSM to design experiments. Under this DoE, the number of experiments can be ascertained as represented in Eq. (4).

$$
N=2^{k}+2 k+c
$$

where $k$ is the independent variables or number of input parameters, $2^{k}$ is the factorial points with $n$ factorial runs, $2 k$ is the axial runs and c stands for the central runs.

Table I. Factors, codes and levels for the CCF experimental design

\begin{tabular}{|c|l|l|l|l|l|}
\hline Variables & \multirow{2}{*}{ Unit } & \multirow{2}{*}{ Symbol } & \multicolumn{3}{|c|}{ Levels } \\
\cline { 4 - 6 } & & & -1 & 0 & 1 \\
\hline$D_{i} / D_{o}$ & - & $X_{1}$ & 0.20 & 0.40 & 0.60 \\
\hline$L$ & $\mathrm{~m}$ & $X_{2}$ & 0.30 & 0.32 & 0.34 \\
\hline$P$ & $\mathrm{~m}$ & $X_{3}$ & 0.12 & 0.16 & 0.20 \\
\hline
\end{tabular}

A total of 17 treatments were performed to determine the effects of the selected geometric variables on the $C_{P}$ achieved by the AST. In this study, the RSM experimental design, ANOVA and the three-dimensional (3D) response surface plots were conducted by $\mathrm{R}$ software.

The $C_{P}$ was numerically determined for all the treatments by using a 3D transient CFD simulation employing ANSY Fluent software. For this purpose, the computational domain resulted to be a parallelogram, whose dimensions were expressed in terms of $\mathrm{D}_{0}$. The length, height and width were $20 \mathrm{D}_{\mathrm{o}}, 5 \mathrm{D}_{\mathrm{o}}$ and $3 \mathrm{D}_{\mathrm{o}}$, respectively. The computational domain was divided into two sub-domains (an inner rotating domain and an outer stationary domain), as illustrated in Fig. 1. The inner domain had a cylindrical shape with a diameter of $1.2 \mathrm{D}_{\mathrm{o}}$ and a length of $4 \mathrm{D}_{\mathrm{o}}$. This subdomain included the AST, which was placed in the centerline of the cylinder. The distance from the inlet to the internal rotating zone was $D_{0}$ and the distance from the outlet to the internal rotating zone was $15 \mathrm{D}_{\mathrm{o}}$ to avoid any influence of these boundaries surfaces. The hydrokinetic turbine center was precisely placed in the middle of the cross-section of the channel at $2.5 \mathrm{D}_{\mathrm{o}}$ from the top surface of the domain. Additionally, $\alpha$ and $\mathrm{D}_{\mathrm{o}}$ were fixed at $60^{\circ}$ and $0.1 \mathrm{~m}$, respectively.
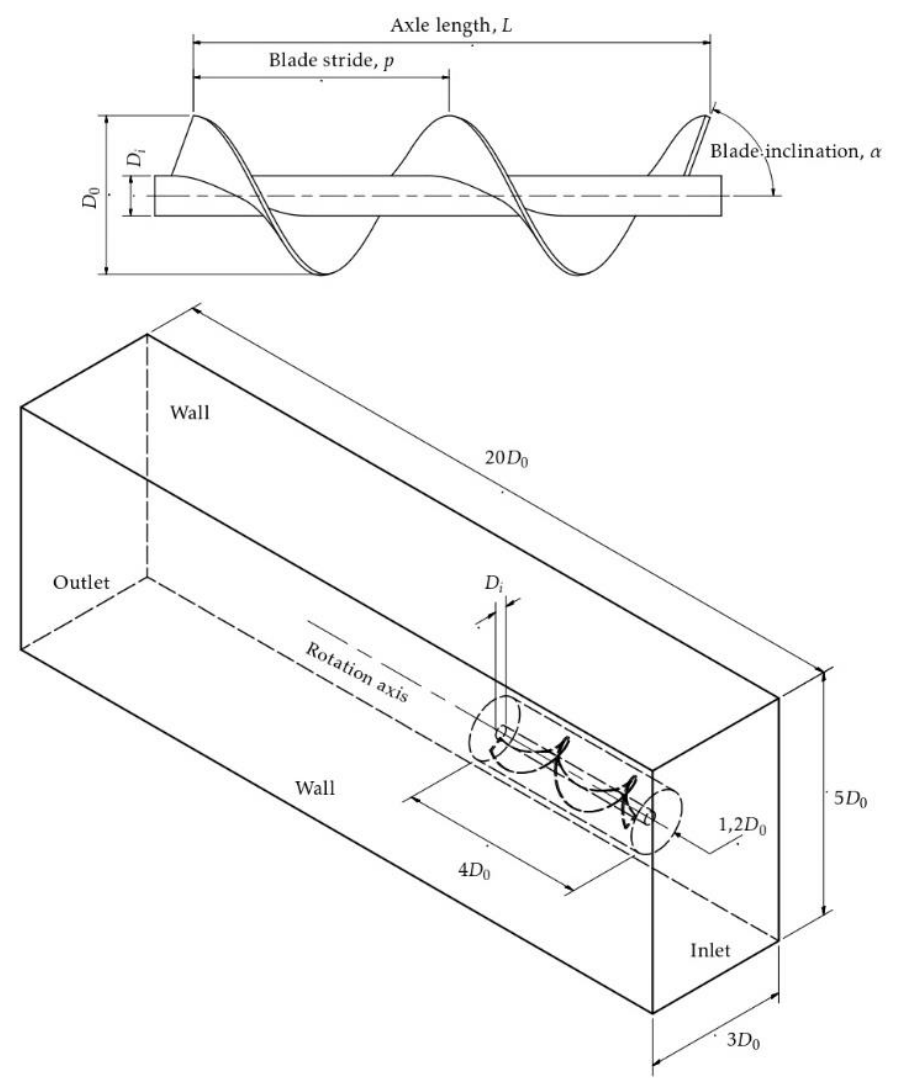

Fig 1. Computational domain in 3D analysis.

The boundary conditions employed in the simulation were the following ones: a uniform velocity inlet boundary applied at the domain right surface. The incoming velocity was $1 \mathrm{~m} / \mathrm{s}$, which is the design velocity. A pressure outlet boundary $(0 \mathrm{~Pa})$ was applied at the domain left surface. No-slip boundary conditions 
were imposed at the surface of the AST and the external walls of the domain. The interfaces between the external stationary subdomain and the rotating subdomain were set as a non-conformal interface. Furthermore, this study used the six-degrees of freedom (6-DoF) UDF function. The 6DoF was chosen since it represents physical phenomena in a more precise way. It is highlighted that the AST rotation is the result of the simulation and it is not a boundary condition. During the simulation of each treatment, a preload and a moment of inertia were defined. The moment of inertia was known by using Computer Aided Design (CAD) software from each AST geometric configuration, which was defined for each run.

The computational domain was discretized by means of a non-structured mesh due to the complexity of the geometry. Using the inflation mesh, the grid was refined so that the value of $y^{+}$near the helical surface was suitable for the shear stress transport (SST) $k-\omega$ turbulence model [10]. It is important to note that the mesh size and the time-step have to be optimized to reduce the calculation costs, while maintaining an acceptable accuracy. The area under the curve conformed by $C_{P}$ vs. TSR was chosen as the benchmark of the mesh quality. According to the mesh independence study using the grid convergence index, the threshold of the mesh was found to be of 409917 elements. Additionally, the results were independent with regard to the time steps for values below $0.001 \mathrm{~s}$.

\section{Results and discussion}

The numerical results regarding the maximal $C_{P}$ obtained from the treatments are presented in Table II. A good agreement can be seen between the CFD results and the predicted values.

The relationship between the AST performance and the TSR for each treatment was presented in Fig. 2. The $C_{P}$ curves were obtained by the CFD simulations.

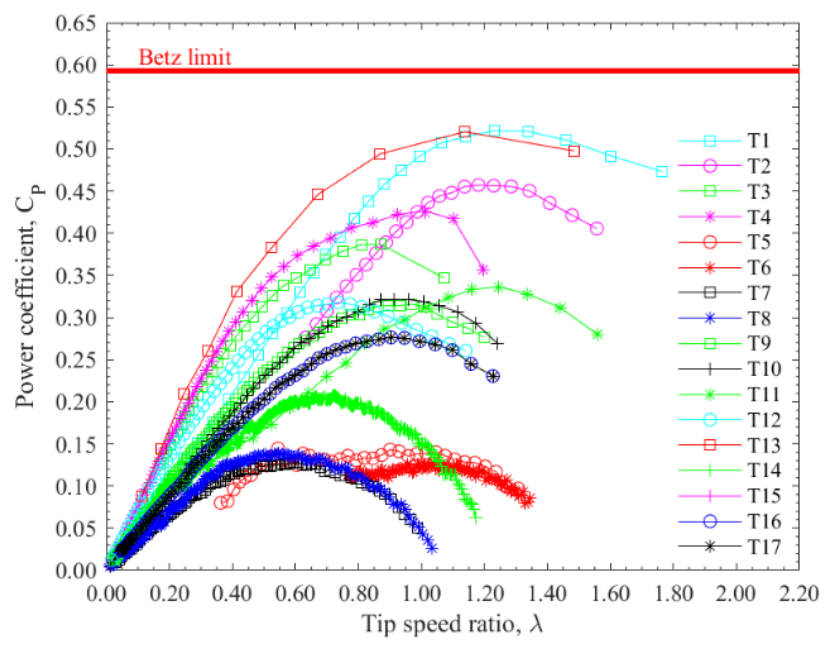

Fig. 2. Power coefficient $\left(C_{P}\right) v s$. tip speed ratio $(\lambda)$ for all the treatments.
Table II. Matrix domain concerning the CCF experimental design

\begin{tabular}{|c|c|c|c|c|c|}
\hline Run & $\begin{array}{c}\text { Axle } \\
\text { length, }\end{array}$ & $\begin{array}{c}\text { Blade } \\
\text { stride, } \\
\end{array}$ & $\begin{array}{c}\text { Diameter } \\
\text { ratio }\end{array}$ & $p(\mathrm{~m})$ & \multicolumn{2}{|c|}{$\begin{array}{c}\text { Maximal power } \\
\text { coefficient }\left(D_{P_{\text {max }}}\right)\end{array}$} \\
\cline { 5 - 6 } & & & $\left.D_{o}\right)$ & $\begin{array}{c}\text { CFD } \\
\text { results }\end{array}$ & $\begin{array}{c}\text { Predicted } \\
\text { results }\end{array}$ \\
\hline 1 & 0.30 & 0.12 & 0.2 & 0.5113 & 0.5063 \\
\hline 2 & 0.34 & 0.12 & 0.2 & 0.4572 & 0.4713 \\
\hline 3 & 0.30 & 0.20 & 0.2 & 0.3865 & 0.3990 \\
\hline 4 & 0.34 & 0.20 & 0.2 & 0.4263 & 0.42435 \\
\hline 5 & 0.30 & 0.12 & 0.6 & 0.1446 & 0.1555 \\
\hline 6 & 0.34 & 0.12 & 0.6 & 0.1286 & 0.1205 \\
\hline 7 & 0.30 & 0.20 & 0.6 & 0.1280 & 0.1229 \\
\hline 8 & 0.34 & 0.20 & 0.6 & 0.1389 & 0.1482 \\
\hline 9 & 0.30 & 0.16 & 0.4 & 0.3154 & 0.3098 \\
\hline 10 & 0.34 & 0.16 & 0.4 & 0.3106 & 0.3049 \\
\hline 11 & 0.32 & 0.12 & 0.4 & 0.3253 & 0.3134 \\
\hline 12 & 0.32 & 0.20 & 0.4 & 0.2883 & 0.2736 \\
\hline 13 & 0.32 & 0.16 & 0.2 & 0.509 & 0.4641 \\
\hline 14 & 0.32 & 0.16 & 0.6 & 0.1831 & 0.1506 \\
\hline 15 & 0.32 & 0.16 & 0.4 & 0.2778 & 0.3074 \\
\hline 16 & 0.32 & 0.16 & 0.4 & 0.2778 & 0.3074 \\
\hline 17 & 0.32 & 0.16 & 0.4 & 0.2778 & 0.3074 \\
\hline
\end{tabular}

The equation for predicting the optimal geometric design configuration of the AST and its effect on the $C_{P}$ was obtained according to the CCF experimental design and the selected input variables. The second-order polynomial model was defined by Eq. (4). Based on the statistical analysis, the determination coefficient $\left(R^{2}\right)$ and the adjusted determination coefficient $\left(R_{a d j}^{2}\right)$ were $97.4 \%$ and $95.8 \%$, respectively. The results indicated that the quadratic regression model built was adequate for representing the response variable regarding the CFD results.

$$
\begin{gathered}
C p=1.6332-3.141 L-4.6998 p-1.1571 D_{i} / D_{o} \\
+18.8750 L * p+2.3344 p \\
* D_{i} / D_{o}-8.6607 p^{2}
\end{gathered}
$$

The ANOVA results were tabulated in Table III. The significance of the regression model equation and the coefficient terms of the model were determined by the $F$ ratio and the $p$-value. Each variable with a $p$-value lower than 0.05 is considered statistically significant; while those ones with a $p$-value higher than 0.05 were nonsignificant from a statistical point of view. The terms $L * D_{i} / D_{o}, L^{2}$ and $\left(D_{i} / D_{o}\right)^{2}$, which resulted to have a larger $p$-value, were excluded from the model. The ANOVA results indicated that the $F$-ratio and the $p$-value for the regression model were 62.55 and $2.368 \times 10^{-7}$, respectively, indicating that the model was significant. Table III. Statistical analysis of variance (ANOVA) 


\begin{tabular}{|l|c|c|c|l|l|}
\hline \multicolumn{1}{|c|}{ Term } & SS* & Df* & MS* & $F$-ratio & $p$-value \\
\hline $\begin{array}{l}\text { Axle length, } \\
L(\mathrm{~m})\end{array}$ & $5.8564 \times 10^{-5}$ & 1 & $5.8564 \times 10^{-5}$ & 0.09 & 0.7751 \\
\hline $\begin{array}{l}\text { Blade stride, } \\
p(\mathrm{~m})\end{array}$ & 0.0039601 & 1 & 0,0039601 & 5.83 & 0.0364 \\
\hline $\begin{array}{l}\text { Diameter } \\
\text { ratio, } D_{i} / D_{o}\end{array}$ & 0.24558 & 1 & 0.24558 & 361.41 & $3.52 \times 10^{-9}$ \\
\hline$L * p$ & 0.00182408 & 1 & 0.00182408 & 2.68 & 0.1324 \\
\hline$p * p$ & 0.00079067 & 1 & 0.00079067 & 1.16 & 0.3060 \\
\hline$p *\left(D_{i} / D_{o}\right)$ & 0.00279004 & 1 & 0.00279004 & 4.11 & 0.0702 \\
\hline Residuals & 0.00679501 & 10 & 0.00067950 & & \\
\hline Total & 0.261799 & 16 & & & \\
\hline *Df: Degrees of freedom; SS: Sum of squares; MS: Mean square. \\
\hline
\end{tabular}

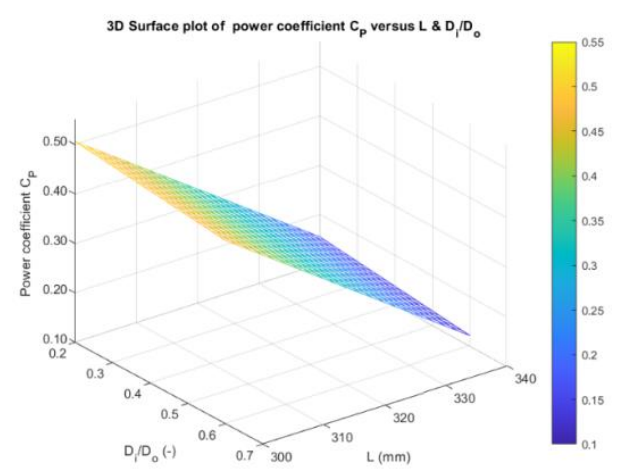

a)

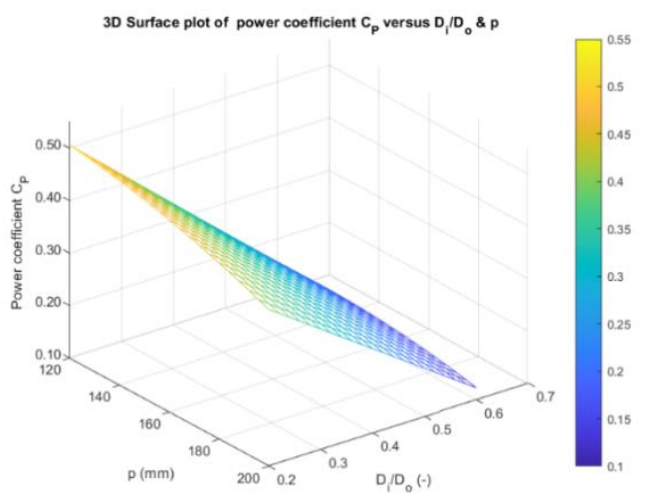

b)

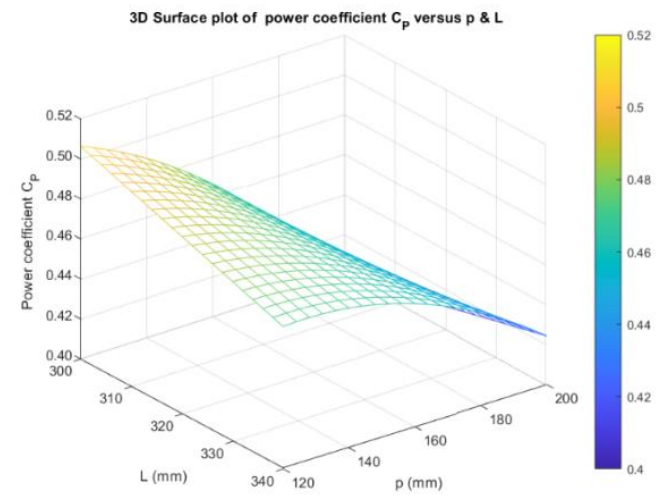

c)

Fig 3. Response surface plots of $C_{P} v s$. the effect of the variables, including a) $L$ and $D_{i} / D_{o}$, b) $P$ and $D_{i} / D_{o}$, c) $L$ and $P$.
The variables $L, L * p, p * p$ and $p *\left(D_{i} / D_{o}\right)$ were nonsignificant parameters, while $D_{i} / D_{o}$ and $p$ were significant factors. It was also found that when $L$ is equal to $0.34 \mathrm{~m}$, the variation of $p$ in the range under study $(0.12-0.16 \mathrm{~m})$ does not significantly affect the response variable; i.e., $C_{p}$. The $3 \mathrm{D}$ response surface plots were represented in Fig. 3. Obviously, $C_{P}$ was sensitive to $D_{i} / D_{o}$, which was consistent with the results analysed in Table III. Each plot shown in Fig. 3 presents the effect of two variables on the $C_{P}$ of the AST at the optimal point of the other variable.

Fig. 3 reveals that when $D_{i} / D_{o}$ decreases, $C_{P}$ is increased. From Eq. (4), the $C_{P \max }$ was calculated and resulted to be 0.5063 when $L, p$, and $D_{i} / D_{o}$ were equal to $0.30 \mathrm{~m}, 0.12 \mathrm{~m}$ and 0.2 , respectively.

Generally, the residuals were calculated from the difference between the CFD results and the predicted values. The assumptions of normal distribution, independence and equal variance of the residuals were checked to validate the quadratic regression model. The results from Shapiro-Wilks, Durbin-Watson and studentized Breusch-Pagan tests were 0.3059, 0.9239 and 0.2571 , respectively.

\section{Conclusion}

The AST performance was evaluated using numerical simulations through ANSYs Fluent software combined with RSM. The use of these statistical techniques facilitated the evaluation of several independent geometric parameters, such as $L, P$ and $D_{i} / D_{o}$, on the response variable $\left(C_{P}\right)$. The significance of the independent variables and their interactions were studied and tested through ANOVA.

A second-order quadratic model was built to predict the responses. The regression analysis results revealed that the most significant factors for the measured response were $D_{i} / D_{o}$ and $p$. With regard to the model terms $L, L *$ $p, p * p$ and $p *\left(D_{i} / D_{o}\right)$ were found to not affect in a significant way the AST $C_{P} . R^{2}$ and $R_{a d j}^{2}$ values for the regression model were found to be $97.4 \%$ and $95.8 \%$, respectively. In turn, the p-value associated with the regression model ( $p$-value lower than 0.05 ) indicated that the second-order regression model was highly significant and reliable at a confidence interval of 95\%. By understanding the interaction between variables is helpful to achieve the largest $C_{P}$. The optimal geometric values for $L, p$, and $D_{i} / D_{o}$ were $0.30 \mathrm{~m}, 0.12 \mathrm{~m}$, and 0.2 , respectively. Under these optimal conditions, the maximal $C_{P}$ was 0.5063 .

The results showed that DoE coupled with RSM was a suitable statistical technique for optimizing the geometric parameter in the design of a AST to maximize the turbine $C_{P}$. 


\section{Acknowledgement}

Authors gratefully acknowledge the financial support provided by Tecnológico de Antioquia-Institución Universitaria and Universidad de Antioquia through the research project "Design and experimental characterization of an Archimedean screw-type hydrokinetic turbine (in Spanish)".

\section{References}

[1] A. YoosefDoost and W.D. Lubitz. "Archimedes Screw Turbines: A Sustainable Development Solution for Green and Renewable Energy Generation-A Review of Potential and Design Procedures", Sustainability (2020). Vol. 12 (18), pp. 7352.

[2] G.L. Zitti, A. Brunoric and M. Brocchinia. "Efficiency evaluation of a ductless Archimedes turbine: Laboratory experiments and numerical simulations", Renewable Energy (2020). Vol. 146, pp. 867-879.

[3] S. Waters and G.A. Aggidis. "Over 2000 years in review: Revival of the Archimedes screw from pump to turbine", Renewable and Sustainable Energy Reviews (2015). Vol. 51, pp. 497-505.

[4] K. Shahverdi, R. Loni, B. Ghobadian, S. Gohari, S. Marofi and E. Bellos. "Numerical Optimization Study of Archimedes Screw Turbine (AST): A case study", Renewable Energy (2020). Vol. 145, pp. 2130-2143.
[5] G. Dellinger, P.A. Garambois, N. Dellinger, M. Dufresne, A. Terfous, J. Vazquez and A. Ghenaim. "Computational fluid dynamics modeling for the design of Archimedes Screw Generator", Renewable Energy (2018). Vol. 118, pp. 847-857.

[6] A. Stergiopoulou, V. Stergiopoulos and E. Kalkani. "Computational Fluid Dynamics study on a 3D graphic solid model of Archimedean screw turbines", Int. Conf. Environ. Manag. Eng. Planning, Econ. (2014). Vol. 23(11), pp. 7-14.

[7] A. Stergiopoulou, V. Stergiopoulos and E. Kalkani. "Experimental and theoretical research of zero head innovative horizontal axis Archimedean screw turbines", International Journal of Energy and Environment (2015). Vol. 6 (5), pp. 471.

[8] A. Stergiopoulou, V. Stergiopoulos and E. Kalkani. "Greece beyond the horizon of the era of transition: Archimedean screw hydropower development terra incognita", International Journal of Energy and Environment (2015). Vol. 6(6). pp. 527.

[9] D.C. Montgomery. Introduction to statistical quality control. USA. John Wiley \& Sons (2019).

[10] F.R. Menter. "Two-equation eddy-viscosity turbulence models for engineering applications", AIAA journal (1994). Vol. 32(8), pp. 1598-1605. 